\title{
An $a b$ initio Hartree-Fock study of the reactions of thiiranes with amines
}

\author{
Harold D. Banks and William E. White \\ U.S. Army Edgewood Chemical Biological Center Aberdeen Proving Ground, MD 21010-5424, \\ USA \\ E-mail: harold.banks@sbccom.apgea.army.mil
}

(received 10 Jan 00; accepted 25 Apr 00; published on the web 03 May 00)

\begin{abstract}
The reactions of thiirane with ammonia, a series of primary amines and aniline have been studied by means of $a b$ initio Hartree-Fock calculations at the $6-31 G^{*}$ level. The transition state geometries were characterized and used to determine the relative rates of reaction. In all cases, anti attack by the nucleophile was favored. Relative rates for the aliphatic amines varied by a factor of less than twenty, with ethylamine being most reactive. Ammonia reacted more than twenty and aniline almost $8 \times 10^{4}$ times slower than tert-butylamine. The results can be rationalized on the basis of relative nucleophilicity and nonbonding interactions.
\end{abstract}

Keywords: Thiiranes, Hartree-Fock calculations, transition state geometries

\section{Introduction}

Thiiranes, ${ }^{1}$ the simplest sulfur heterocycles, occur in nature, and have been used advantageously in the pharmaceutical, polymer, pesticide and herbicide industries. ${ }^{2}$ Very recently the utility of thiirane intermediates was demonstrated in a synthesis of thioglycosides. ${ }^{3}$ Their oxygen analogs, epoxides, have enjoyed numerous synthetic applications due in part to their relative ease of handling, and reactivity with a range of nucleophiles with predictable regio- and stereoselectivity. Thiiranes offer these synthetic possibilities combined with the rich chemistry that a vicinal thiol group in the product provides. Extensive development of this chemistry of thiiranes has been thwarted in part by the observation that yields of nucleophilic substitution products can be significantly lowered by polymerization side reactions occasioned by the generation of nucleophilic products that compete for unreacted thiirane. In addition, certain physical properties of low molecular weight sulfur compounds have diminished their attractiveness to potential practitioners. ${ }^{4}$ Given the dearth of research on the relative rates of nucleophilic opening reactions of thiiranes, ${ }^{5}$ the following investigation was undertaken.

This study calculated the relative rates of reaction of the parent compound with ammonia, a series of primary aliphatic amines and aniline. One advantage of computational chemistry is that 
it can significantly reduce the number of laboratory experiments that have to be performed by focusing on those key reactions that calculation identifies as most promising. It is assumed that the most reactive amines are most likely to provide acceptable yields of substitution products. Ideally, synergism between the laboratory and computer can result in expeditious solutions to synthetic problems. While most synthetic reactions are performed in solution, our initial calculations were performed in the absence of solvent. Gaussian $94^{6}$ was used to identify the transition state, its geometry and corresponding thermodynamic parameters. Transition state theory was then applied in order to determine the relative rates of the reaction.

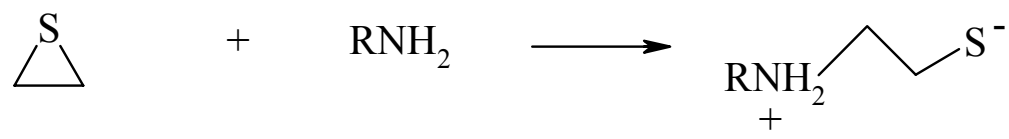

Calculations for ammonia, possessing $\mathrm{C}_{3 \mathrm{v}}$ symmetry, were straightforward. For the remaining amines of lower symmetry classification, molecular mechanics was used to identify each conformational minimum. In order to increase the likelihood of discovering all available transition states, three modes of initial approach were considered for each conformation. These were generated by first choosing a staggered orientation about the forming carbon-nitrogen bond; the remaining two approaches resulted from rotation about this partial bond by increments of $120^{\circ}$. The relative free energies of the conformations allowed their mole fractions to be determined and used in calculation of the total rate of the amine. ${ }^{7}$ It is interesting to note that the approach in which the alkyl group of the amine assumed an anti orientation about the forming carbon-nitrogen bond with respect to the carbon-carbon bond of the thiirane was invariably found to be the lowest energy pathway.The Figure provides the transition state geometry for the anti mode (left) and for one of the gauche modes of approach for the reaction of methylamine with thiirane. Careful inspection of the second approach will demonstrate that the dihedral angle of the C-N---C-C bond is larger than $120^{\circ}$. This is an apparent attempt of the transition state structure to minimize unfavorable nonbonding interactions between the amine methyl and the distant methylene group of the thiirane.
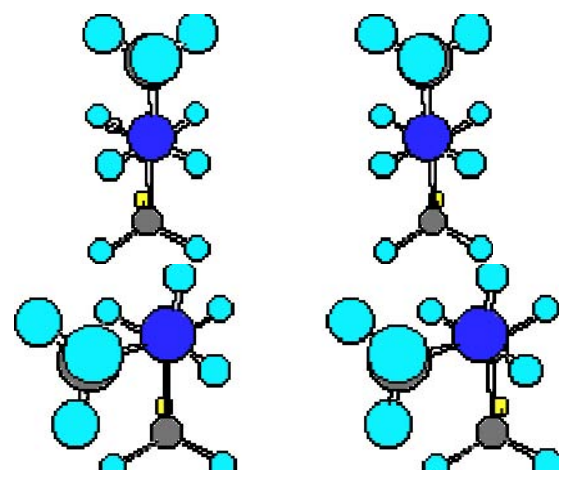

Figure 1. Stereoviews for transition states resulting from two modes of approach of methylamine to thiirane. 
Each unique approach for every conformation for a given amine resulted in a specific reaction rate. Analysis of these data was accomplished by means of Curtin-Hammett/Winstein-Holness kinetics. ${ }^{8,9}$ The rates of these parallel reactions for each conformation were summed, multiplied by the conformational mole fraction and added to these quantities derived from the other conformations to give the total rate.Calculations for certain amines were rather lengthy. Ethylamine and isopropylamine were found to have two and four conformational minima respectively.A total of six transition states were incorporated into the calculation for the former amine, and twelve for the latter.

In the case of tert-butylamine, only one transition state corresponding to an anti orientation was derived regardless of the geometry of the initial approach. This finding emphasizes the importance of nonbonding interactions in the transition state.

The Table provides the relative rates of reaction for ammonia and primary amines with thiirane. Primary aliphatic amines should be considerably more reactive than ammonia. Ethylamine is predicted to be the most reactive species. This can be rationalized by the observation that it represents the best compromise between nucleophilicity and steric bulk. When the size of the attached alkyl group becomes important as is the case for isopropyl and tert-butylamine, the reaction rate is retarded. It is interesting, however, that all aliphatic amines react at reasonable rates with thiirane, the fastest less than twenty times faster than the slowest.

Table 1. Relative Rates of Reaction of Ammonia and Primary Amines with Thiirane at $298.15^{\circ} \mathrm{K}$ in the Gas Phase

\begin{tabular}{ll}
$\mathrm{RNH}_{2}, \mathrm{R}=$ & Relative Rate \\
\hline $\mathrm{H}$ & 0.04437 \\
$\mathrm{CH}_{3}$ & 7.894 \\
$\mathrm{CH}_{3} \mathrm{CH}_{2}$ & 16.940 \\
$\left(\mathrm{CH}_{3}\right)_{2} \mathrm{CH}$ & 1.227 \\
$\left(\mathrm{CH}_{3}\right)_{3} \mathrm{C}$ & 1.000 \\
$\mathrm{C}_{6} \mathrm{H}_{5}$ & $7.946 \times 10^{-4}$
\end{tabular}

As expected, aniline is the least reactive nucleophile. Such behavior is typical of aromatic amines. ${ }^{10}$

As mentioned above, there is scant experimental data for comparison. While use of reaction yields to assess relative reaction rates is fraught with difficulties (differences in solvent, temperature, reaction times, skill in product isolation), these data represent the only link between theory and experiment. When thiirane was reacted in a sealed tube at $90-100^{\circ}$, the yields with primary amines were ca. $50 \%$ following purification by distillation; the yield with aniline was $52 \%{ }^{11}$ Using ethylene monothiolcarbonate, ${ }^{12}$ 2-thioethyl carbamates, ${ }^{13}$ and 2-hydroxyethyl- 
thiolcarbonates ${ }^{14-16}$ in the presence of base for the in situ generation of thiirane, Reynolds and coworkers found aniline to be inert in refluxing toluene, conditions that produced good yields with aliphatic amines. These results are in qualitative agreement with those obtained computationally; clearly the results for aniline are divergent ${ }^{17}$ and should be reinvestigated.

The intrinsic relative reactivities of thiirane and oxirane, the compound routinely chosen for synthesis of $\beta$-substituted alcohols, were calculated. With both ammonia and methylamine, the sulfur heterocycle was calculated to react more than $10^{13}$ times faster than its oxygen analogue in the gas phase. This rather surprising observation appears to be at odds with laboratory experience. It is likely that protons, proton sources or other acid species are present in most experiments in sufficient quantity to catalyze the oxirane opening reaction.

It should be borne in mind that these calculations of predicted reactivity for thiirane with selected nucleophiles were conducted in the gas phase. This approach was taken because of the fact that in many cases, when similar systems are compared, reasonable agreement between gas phase calculations and the experimental results is obtained. From a practical point of view, the calculation time is considerably diminished when solvent models ${ }^{18}$ are not included. Once the geometry of the gas phase transition state is obtained, it may be used as the starting point for solution calculations, leading to a reduction in computational time.

Most synthetic reactions are conducted in solution. Because the reaction of thiirane with amines results in a zwitterionic product with charge development reflected in the transition state, one would anticipate the solvent to play an important role in reaction rates. More polar solvents would be expected to stabilize the transition state and accelerate the reaction. To obtain a qualitative concept of how the rates might be affected in solution, the dipole moments were obtained from the output files. Thiirane is rather nonpolar with a calculated dipole moment, $\mu$, of 0.8633 Debye, lower than the experimental value. ${ }^{19 a}$ In its reaction with ammonia,

$\mu=1.7768 \mathrm{D},{ }^{19 \mathrm{~b}}$ the dipole moment increases significantly to $10.7274 \mathrm{D}$. A similar result is obtained for the aliphatic amines with calculated $\mu_{\mathrm{ave}}=1.4238 \mathrm{D}$ transformed into transition states with calculated $\mu_{\text {ave }}=10.9202 \mathrm{D}$. The present calculations demonstrate that there is a considerable increase in polarity of the transition state. Given the ability of an aromatic ring to accept positive charge, it is not surprising that the largest change in polarity occurs in the reaction of aniline. Specifically, computations for aniline give the following results: $\mu=1.6090$ $\mathrm{D},{ }^{19 \mathrm{c}}$ while that of its transition state was determined to be $12.9000 \mathrm{D}$. Since solvent effects can drastically alter nucleophilicity, ${ }^{20}$ computational methods provide a rational approach to prediction of rate changes in solution. Such calculations are in progress and will be reported in due course.

\section{Computational Methodology}

All calculations were performed on a Silicon Graphics Octane workstation. Molecular mechanics conformational searches were conducted using MacroModel Version 6.0. ${ }^{21}$ Gaussian $94^{6}$ was 
used for the restricted Hartree-Fock calculations using the $6-31 \mathrm{G}^{*}$ basis set $\left(\mathrm{HF} / 6-31 \mathrm{G}^{*}\right)$. To assure a thorough search for the transition state, the keyword, calcall, was included in the route section of the input file. The starting geometry for a chosen approach for a particular conformation was obtained using the semi-empirical AM1 method available in Spartan Version 5.1.1 $1^{22}$ to conduct a transition structure search. The Cartesian coordinates so obtained were converted to a Z-matrix by means of the newzmat utility and inserted into the input file. Optimization of the ground states for the reactants included a frequency calculation so that the thermodynamic parameters would be computed. The primary requirement for successful location of a transition state was finding only one imaginary frequency. Animation of this frequency using GaussView demonstrated whether this frequency was reasonably placed along the reaction coordinate. Alternatively the reaction path protocol (IRC keyword) could be employed. A scale factor of 0.9135 was used for the zero point energies to eliminate systematic errors.

\section{References}

1. For reviews, see (a) Dittmer, D.C.; Thiiranes and Thiirenes In Comprehensive Heterocyclic Chemistry, Vol. 7, Katritzky, A.R.; Rees, C.W., Eds., Pergamon Press: Elmsford, NY, 1984, p 132. (b) Kamal, J. In Second Supplement to the $2^{\text {nd }}$ Edition of Rodd's Chemistry of Carbon Compounds, Vol. IV A, Sainsbury, A., Ed., Elsevier Science B.V.: Amsterdam, 1997, p 49. (c) Fokin, A.V.; Kolomiets, A.F. Uspekhi Khimii, 1976, 45, 71. (d) Reynolds, D.D.; Fields, D.L. In The Chemistry of Heterocyclic Compounds, Vol. 19, Part 1, Weissberger, A., Ed., John Wiley and Sons, Inc.: New York, 1964, p 576.

2. Ref. 1a, pp. 182-184

3. Knapp, S.; Malolanarasimhan, K. Org. Lett., 1999, 1, 611

4. Block, E. Reactions of Organosulfur Compounds; Academic Press: New York, NY, 1978, pp 2-3

5. Han, I-S.; Kin, D.K.; Kim, D.K.; Lee, B-S.; Lee, I. J. Comput. Chem., 1997, 18, 1773. This paper reports the results of calculations for the reverse reaction, intramolecular displacement of fluoride using 2-fluoroethanethiolate.

6. Frisch, M.J.; Trucks, G.W.; Schlegel, H.B.; Gill, P.M.W.; Johnson, B.G.; Robb, M.A.; Cheeseman, J.R.; Keith, T.; Petersson, G.A; Montgomery, J.A.; Raghavachari, K.; AlLaham, M.A.; Zakrzewski, V.G.; Ortiz, J.V.; Foresman, J.B.; Cioslowski, J.; Stefanov, B.B.; Nanayakkara, A.; Challancombe, M.; Peng, C.Y.; Ayala, P.Y.; Chen, W.; Wong, M.W.; Andres, J.L.; Replogle, E.S.; Gomperts, R.; Martin, R.L.; Fox, D.J.; Binkley, J.S.; Defrees, D.J.; Baker, J.; Stewart, J.P.; Head-Gordon, M.; Gonzolez, C.; Pople, J.A Gaussian 94, Gaussian, Inc., Pittsburgh, PA, 1995.

7. Eliel, E.L.; Wilen, S.H. Stereochemistry of Organic Compounds, John Wiley and Sons, Inc.: New York, 1994, pp 629-634

8. Seeman, J.I. Chem. Rev., 1983, 83, 84. 
9. Eliel, E.L. J. Chem. Ed., 1975, 52, 762.

10. Ref 11a, p 233; Ref. 7b, pp 333-334.

11. Snyder, H.R.; Stewart, J.M.; Ziegler, J.B. J. Am. Chem. Soc., 1947, 69, 2672.

12. Reynolds, D.D.; Massad, M.K.; Fields,Johnson, D.L. J. Org. Chem., 1961, 26, 5109. (b) Reynolds, D.D.; Fields,Johnson, D.L.J. Org. Chem., 1961, 26, 5111.

13. Reynolds, D.D.; Fields,Johnson, D.L. J. Org. Chem., 1961, 26, 5116.

14. Reynolds, D.D.; Fields,Johnson, D.L. J. Org. Chem., 1961, 26, 5119.

15. Reynolds, D.D.; Fields,Johnson, D.L. J. Org. Chem., 1961, 26, 5122.

16. Reynolds, D.D.; Fields,Johnson, D.L. J. Org. Chem., 1961, 26, 5125.

17. Braz, G.I. J. Gen. Chem. U. S. S. R., 1951, 21, 757. The reaction of aniline with thiirane is reported to occur in good yield after five days at room temperature.

18. Cramer, C.J.; Truhlar, D.G. Chem. Rev., 1999, 99, 2161.

19. The experimental dipole moments are: (a) Cunningham, Jr.,G.L.; Boyd, A.W.; Myers, R.J.; Gwinn, W.D.; Le Van, W.I. J. Chem. Phys., 1951, 19, 676. 1.84 D (gas phase.)

20. Hellwege, K.H., Ed. Relationships in Science and Technology, Group II, Vol. 14a, SpringerVerlag: Heidelberg, 1982. 1.47 D (gas phase.) (c) Gross, P. Physik. Z., 1931, 32, 17821784. 1.49 D (gas phase.)

21. Pross, A. Theoretical and Physical Principles of Organic Chemistry, JohnWiley and Sons, Inc.: New York, 1995, pp 239-240. (b) Lowry, T.H.; Richardson, K.S. Mechanism and Theory in Organic Chemistry, $2^{\text {nd }}$ Edn, Harper and Row: New York, p 323. (c) March, J. Advanced Organic Chemistry, $4^{\text {th }}$ Edn, John Wiley and Sons: New York, 1992, 357.

22. Mohamadi, F.; Richards, N.G.J.; Guida, W.C.; Liskamp, R.; Lipton, M.; Caulfield, C.; Chang, F., Hendrickson, T.; Still, W.C. J. Comput. Chem., 1990, 11, 440.

23. Spartan Version 5.5.1: Wavefunction Inc., 18401 Von Karman Avenue, Suite 370, Irvine, CA 92612. 